\title{
Optical properties of excitons in GaAs quantum wires
}

\author{
R. RINALDI, R. CINGOLANI ${ }^{(1)}$, M. FERRARA, U. MARTI ${ }^{*}$, D. MARTIN ${ }^{*}$, F.K. REINHART ${ }^{*}$, \\ H. LAGE ${ }^{* *}$, D. HEITMANN** and K. PLOOG**
}

Unità GNEQP, Dipartimento di Fisica, Università di Bari, 70100 Bari, Italy

* Ecole Polytechnique Fédérale de Lausanne, Lausanne, Switzerland

${ }^{* *}$ Max-Planck-Institut für Festkörperforschung, 7000 Stuttgart 80, Germany

\begin{abstract}
We report the result of a detailed spectroscopic investigation of the optical properties of excitons in rectangular (etched) and V-shaped (grown on non-planar substrates) GaAs quantum wires. High index excitonic transitions and strong polarization anisotropy of the optical spectra have been observed. The experimentally observed trasitions are in agreement with the theoretical evaluation of quantized energy levels based on the respective model potential.
\end{abstract}

\section{Introduction}

The primary aim of the fundamental and applied research in the field of low dimensional semiconductors is the realization of quantum wires and quantum dots of high optical quality, i.e., with high emission efficiency and strong non-linear behavior. Unfortunately, the optical properties of the available quantum wires heterostructures are still strongly dependent on the fabrication technology. In this work we discuss the excitonic properties of quantum wires as a function of their geometrical and physical characteristics.

\section{Experimental}

Two set of quantum wire samples were investigated : a) rectangular free standing quantum wires (RWRs), and b) V-shaped buried quantum wires of triangular section (TWRs). The rectangular quantum wires were obtained by holographic photolithography and plasma etching. Two different RWR samples were investigated : sample A consisting of three etched GaAs quantum wells of nominal thickness $L_{z}=10.6 \mathrm{~nm}$ with $A l_{.30} G a_{.70} A s$ barriers and lateral crystalline width $L_{y} \sim 70 \mathrm{~nm}[1]$, and sample B consisting of $25 \mathrm{GaAs}$ quantum well with the same $L_{z}$ and barrier composition of sample $A$, but grown on an AlGaAs cladding layer providing efficient optical confinement. The effective crystalline width was $\sim 60 \mathrm{~nm}[2]$. The $V$-shaped quantum wires were obtained by growing by MBE a $4 \mathrm{~nm} \mathrm{GaAs}$ quantum well with $(\mathrm{GaAs})_{8}(\mathrm{AlAs})_{4}$ superlattice (SL) barriers on a GaAs non-planar substrate. The substrate was patterned and etched by holographic lithography (U.V He-Cd laser line) and wet chemical etching, resulting in the formation of $250 \mathrm{~nm}$ deep and $250 \mathrm{~nm}$ wide V-shaped grooves. From TEM observations the bent GaAs quantum well was found to be tapered, with thickness varying from about $10 \mathrm{~nm}$ (bottom of the $\mathrm{V}$-groove) to $2.5 \mathrm{~nm}$ (on top of the sidewalls). Two TWRs were investigated: one with a cap layer of AlGaAs (sample C) and the other without cladding layer (sample D). Calculation performed on these structures by means of the method reported in Ref. $\{3\}$ allows to estimate an effective wire width of about $22 \mathrm{~nm}$.

(1) Permanent address: Dipartimento Scienza dei Materiali, Università di Lecce, via Arnesano, 73100 Lecce, Italy 


\section{Results and Discussion}

In rectangular quantum wires, the spectrum of quantized states roughly scales as

$$
E_{n_{x}\left(n_{y}\right)}^{c o n f}=\frac{\hbar^{2}}{2 m^{*}} \cdot\left(\frac{n_{x}\left(n_{y}\right)}{L_{z}}\right)^{2} .
$$

The V-shaped quantum wires are formed during the epitaxial growth at the bottom of an etched groove, due to the preferential growth along the (100) surfaces [4]. This allows the formation of thin $\left(L_{y} \sim 20 \mathrm{~nm}\right)$ triangular quantum wires with smooth interfaces. The carriers confined in the $V$-shaped wires experience a rectangular quantum well confining potential along the $z$-direction and a parabolic-like confining potential $\left(1 / \cosh ^{2}(y)\right.$ profile) along the $y$-direction. This results in a set of almost equally spaced quantum states.

It is interesting to investigate how the lateral potential shape affects the optical properties of excitons confined in the wires. To detect one-dimensional excitonic subbands, temperature and polarization dependent photoluminescence (PL) and photoluminescence excitation (PLE) experiments have been performed. In Fig. Ia the polarization dependent PLE spectra of sample $A$ are reported. In the p-polarized spectrum $\{6\}$ a broad peak around $797.5 \mathrm{~nm}$ and a more intense peak around $793.3 \mathrm{~nm}$ can be observed, while in the s-polarized spectrum only the peak around $797.5 \mathrm{~nm}$ can be recognized. The weighted difference between the normalized s-polarized and p-polarized PLE spectra is plotted in Fig.1b (linear polarization anisotropy ratio). The observed strong polarization anisotropy is consistent with the theoretical predictions: the $n=1$ heavy-hole-like state is stronger when the exciting polarization is parallel to the quantum wires, whereas the light- hole-like state takes advantage of the perpendicular polarization. [7].

As pointed out in Ref.[8], in wide rectangular quantum wires, where the lateral dimension $L_{y}$ is much larger than the exciton Bohr radius $\left(a_{0}\right)$, the lateral extension of the potential is not sufficent to confine the individual carriers. In this case, the exciton wavefunction is confined as a whole by the lateral boundaries, giving rise to the so called "center of mass quantization" (c.m.) of the exciton motion. The dispersion of these center of mass quantized states is

$$
E_{c . m .}^{e x}=1.5545+\frac{\pi^{2} \hbar^{2}}{2 M_{e x}^{2}} \cdot \frac{1}{L_{y}^{2}} \cdot n_{c . m}^{2}
$$

where $M_{e x}^{*}=m_{e}^{*}+m_{h}^{*}$. Taking into account an effective lateral width $L_{y} \simeq 70 \div 80 \mathrm{~nm}$, to account for the dead layer, the c.m. confined heavy hole exciton states are separated in energy by $.42 \div .32 * n_{c . m}^{2} m e V$ with $n_{\text {c.m. }}=1,2,3 \ldots$. Therefore they cannot be resolved within the inhomogeneous broadening of the excitonic peak $(\sim 7 \mathrm{meV})$. The radiative recombination processes between c.m. quantized states can be resolved when the lateral wire dimension is reduced to $60 \mathrm{~nm}$, that is $L_{y} \sim 4 a_{0}$. In this case the PLE spectrum exhibits distinct peaks that can be assigned to radiative recombination processes between c.m. quantized states, as shown in Fig.2 [8]. A strong proof of the existence of c.m. quantized states can be obtained by observing the thermal filling of exciton c.m. states with increasing the temperature (Fig.2). The deconvolution of the temperature dependent PL spectra gives energy separation between peaks in agreement with eq.(2) and a temperature filling factor which follows the Bose-Einstein distribution function

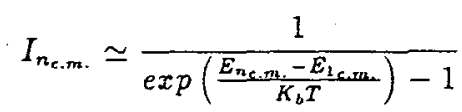

We now turn to the optical properties of the V-shaped quantum wire samples. In Fig. 3 the PLE and temperature dependent PL spectra of sample $C$ are reported. The PLE spectrum exhibits $a \sim 10 \mathrm{meV}$ Stokes Shift due to well width fluctuations and interface roughness, and 
three clearly resolved bumps around $790 \mathrm{~nm}, 784 \mathrm{~nm}$ and $775 \mathrm{~nm}$. The energy spacing between these broad structures is in agreement with the theoretical evaluation of the quantum states in the parabolic-like potential of these TWRs. The individual $n_{y}=1,2,3 \ldots$ excitonic transitions can be distinguished in the temperature dependent PL spectra. The broad structures in the PLE spectrum can be associated with a $1 D$ density of states with a inhomogeneous broadening equivalent to $2 \mathrm{~nm}$ fluctuation in the wire width [9]. The density of states in the wire is very small, and it is possible to observe the band filling of the higher energy states even by slightly increasing the excitation intensity or the temperature under $\mathrm{cw}$ excitation. In the investigated samples the 1D conduction band states are almost equally spaced by about $14 \mathrm{meV}$ ( the height of the parabolic-like barrier is about $135 \mathrm{meV}$ ), therefore the $n_{y} \geq 4$ states become quasiresonant with the quantized states of the lateral quantum well [5], giving a strong increase of the absorption at $770 \mathrm{~nm}$ in the PLE spectrum.

In Fig.4 the PL spectra of sample D excited by a p-polarized and s-polarized Argon laser $\left(\lambda_{\text {exc }}=514.5 \mathrm{~nm}\right.$ ) are reported. The ratio between the $n_{y}=1$ related transition peak (with a strong heavy hole character in the valence band [7]) and the $n_{y}=2$ peak (with a mixed heavylight hole character in the valence band) decreases by $30 \%$ changing the polarization direction from parallel to perpendicular to the wires direction. The small red-shift and the increase of emission intensity in the p-polarized PL spectrum could be due to some $\Delta n \neq 0$ transitions contributing to the first peak. In the inset of Fig.4 the angle dependence of the polarization anisotropy for the investigate TQWR samples is reported. The difference in anisotropy between the two samples is due to the presence of the AIGaAs cap layer in sample $C$, whose radiation absorption prevents the high filling of the $1 \mathrm{D}$ subbands, making necessary a deconvolution of the spectra due to the line broadening $(\sim 11 \mathrm{meV})$. Our results are in agreement with the recent theoretical calculation of Ref.[7], indicating the quite good quality of these samples and the advantage of the growth on non-planar substrates for the fabrication of narrow quantum wires. The observed strong polarization dependence of the PL indicates a strong anysotropy of the optical matrix elements in our samples. Part of this anysotropy cames from the ID confinement, whereas contributions from exstrinsic effects like strain or state mixing due to non-planar slope of the wires cannot be excluded at present.

In conclusion, we have investigated the optical properties of excitons confined in two directions in rectangular and triangular quantum wires, with respect to the shape of their respective confining potentials. This work was partially supported by the Esprit NANOPT project.

\section{References}

[1] L.Tapfer private communication

[2] L.Tapfer, G.C.LaRocca, H.Lage, R.Cingolani, P.Grambow, A.Fisher, D.Heitmann, and K.Ploog, Surf. Sci. 267,227 (1992)

[3] E.Kapon, D.M.Hwang, and R.Bhat, Phys. Rev. Lett. 63, 430 (1989)

[4] R.Bhat, E.Kapon, D.M.Hwang, M.A.Koza, and C.P.Yun, J. Chrystal Growth 93, 850 (1988)

[5] E.Kapon, K.Kash, E.M.Clauser, D.M.Hwang, and E.Colas, Appl. Phys. Lett. 60, 477 (1992)

[6] The s-polarization is achieved when the electric field polarization vector is parallel to the wire direction, while the p-polarization refers to elctric field polarization vector perpendicular and in the same plane of the quantum wires

[7] U.Bockelmann and G.Bastard, Phys Rev. B45, 1688 (1992)

D.S.Citrin and Yia-Chung Chang, Phys. Rev. B43, 11703 (1990)

[8] H.Lage, D.Heitmann, R.Cingolani, P.Grambow, and K.Ploog, Phys. Rev. B44, 6550 (1991)

R.Rinaldi, R.Cingolani, M.Ferrara, H.Lage, D.Heitmann, and K.Ploog, Phys. Rev. B47, 7275 (1993)

[9] H.Zarem, K.Vahala, and A.Yariv, IEEE J. Quantum Electron. QE-25, 705 (1989) 

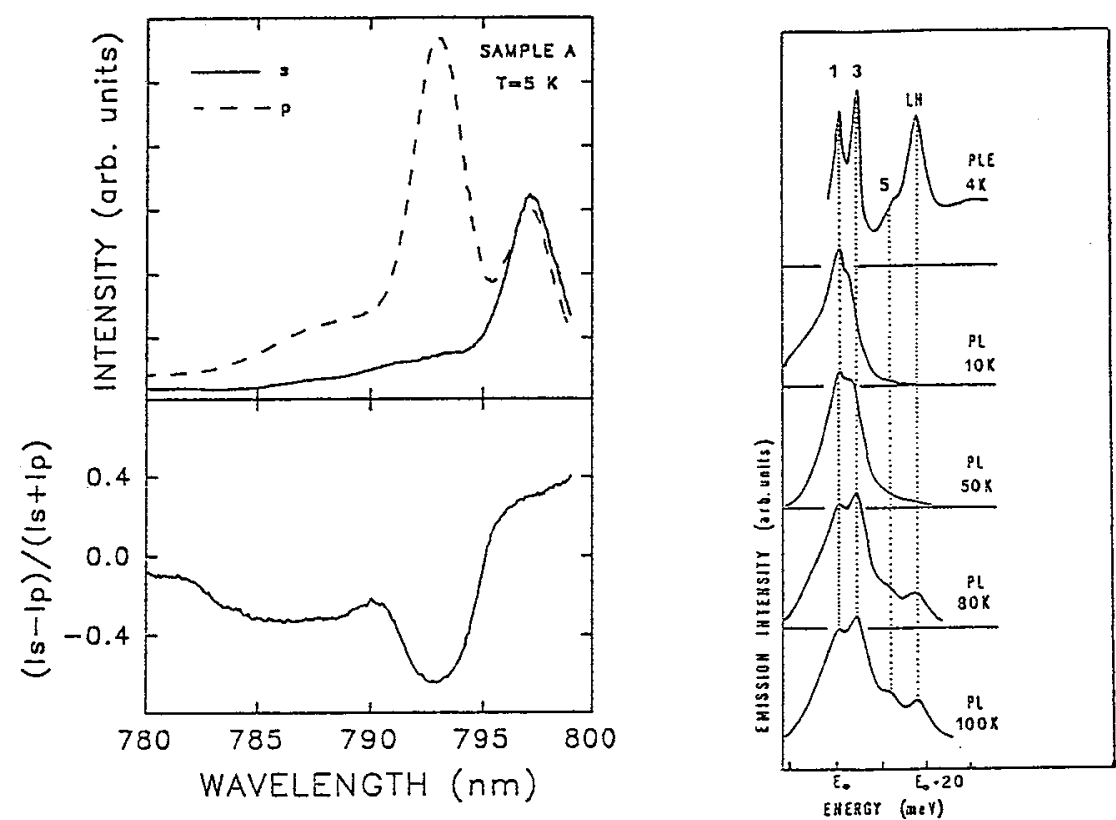

Fig.1 - a) p-and s-polarized PLE spectra of sample A. The detection wavelength was set to $800 \mathrm{~nm}$. b) weighted polarization anisotropy of PLE spectra reported in Fig.2a)

Fig. 2 - PLE and temperature dependent PL spectra of sample B. $E_{0}=1.5545 \mathrm{eV}$
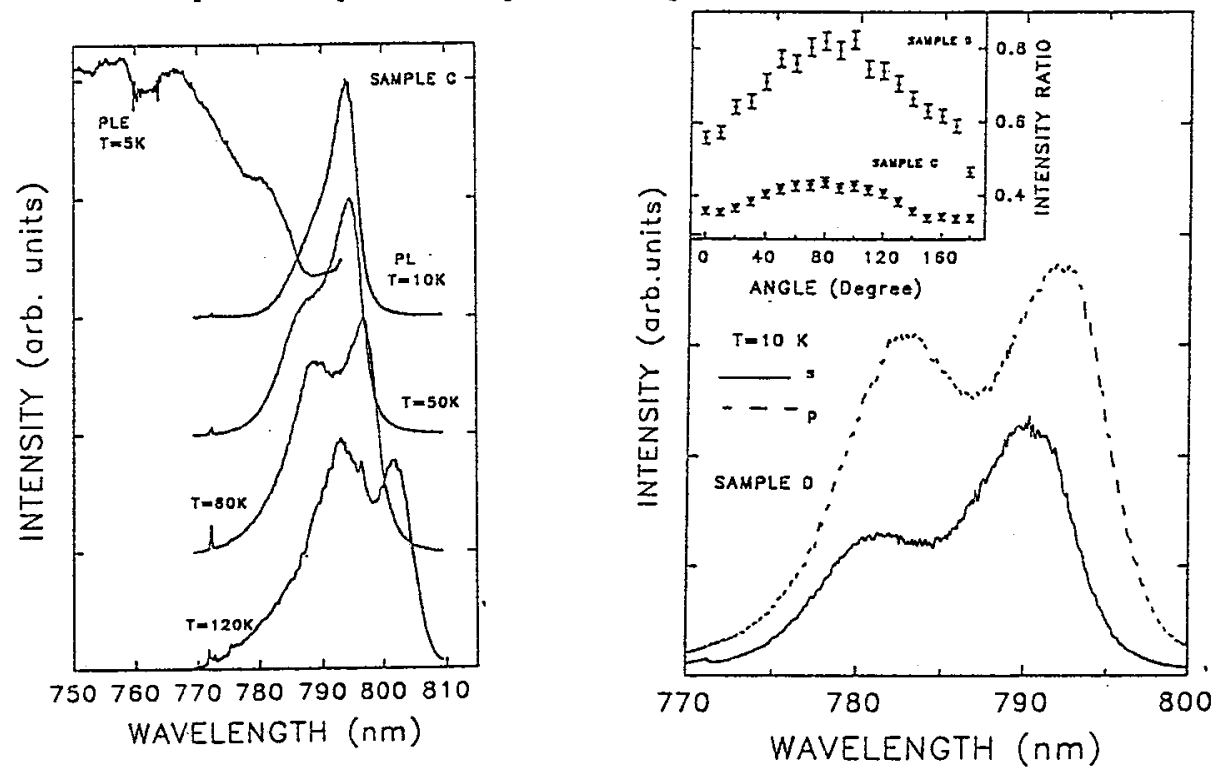

Fig.3 - PLE and temperature dependent PL $\left(I_{e x c}=3 \mathrm{~W} \mathrm{~cm}^{-2}\right.$ - HeNe laser $)$ spectra of sample C. The PLE detection wavelength was set to $794 \mathrm{~nm}$.

Fig.4 - Polarization dependent PL spectra of sample $D\left(I_{e x c}=10 \mathrm{~W} \mathrm{~cm}^{-2}\right.$ - Argon laser). - inset: polarization angle dependence of the intensity ratio $I_{n_{y}=2} / I_{n_{y}=1}$ between the first two excitonic peaks in the PL spectra of sample C and $\mathrm{D}$. 